\title{
DESIGN OF A SIMPLIFIED 3D-PRINTED ARTIFICIAL UNDERACTUATED HAND
}

\author{
A. Orabona, A. Palazzi, S. Graziosi ${ }^{凶}$, F. Ferrise and M. Bordegoni \\ Politecnico di Milano, Italy \\ $\triangle$ serena.graziosi@polimi.it
}

\begin{abstract}
The recent interest in human-robot interaction requires the development of new gripping solutions, compared to those already available and widely used. One of the most advanced solutions in nature is that of the human hand, and several research contributions try to replicate its functionality. Technological advances in manufacturing technologies and design tools are opening possibilities in the design of new solutions. The paper reports the results of the design of an underactuated artificial robotic hand, designed by exploiting the benefits offered by additive manufacturing technologies.
\end{abstract}

Keywords: additive manufacturing, mechatronics, 3D printing, design for additive manufacturing

\section{Introduction}

In years, artificial hands have been designed in multiple variants, to fulfil the design requirements and goals of a wide range of application domains ranging from assistive robotics and prosthetics to autonomous manipulation and logistics (Piazza et al., 2019). Besides, these design requirements and goals are also evolving continuously. For example, the design of grippers used in autonomous manipulation tasks was in the past mainly driven by the need for robustness and safety; nowadays, solutions able to adapt themselves to external and unstructured environments and to interact with humans are needed (Piazza et al., 2019; Bhatia et al., 2019). Indeed, the Industry 4.0 paradigm is actively promoting human-robot collaborations in production lines (Matsas et al., 2018). Standard industrial grippers usually employ a two- or three-point pinch grasp, which is thus limited compared to humans' grasping capability (Kappassov et al., 2013). Hence, the possibility to have grippers able to mimic the appearance and the mechanics of human's hands represents a step forward towards multiple targets. Improved functionalities and anthropomorphic appearance are requirements expected also for prostheses (Ten Kate et al., 2017). Both these two applications domains, despite their intrinsic differences, share also the need for cheap and less complicated solutions in terms of design and control (Ten Kate et al., 2017; Piazza et al., 2019). Additive Manufacturing (AM) technologies together with the continuous development and miniaturizations of hardware components, and the availability of open-source hardware (Piazza et al., 2019), are playing a fundamental role in the evolution of artificial hands. 3D-printed robotic hands and 3D-printed soft robotic solutions (Truby et al., 2019; Piazza et al., 2019) are two emerging trends in this field. AM technologies have contributed to reducing the complexity and production efforts of these robotic devices (Tian et al., 2017) enabling, for example, a reduction in the overall number of parts. Cutting-edge examples of 4D printing grippers have also been developed (Ge et al., 2016). Their functionalities are due to the intrinsic properties of shape 
memory polymers used for manufacturing them (Ge et al., 2016), rather than on the actuation, and to the novel 4D printing approach developed by the authors. Such an approach has allowed them designing and printing proofs-of-concept of innovative multimaterial grippers. The combination of materials properties, such as the ones of elastomers, with AM technologies, has also been successfully exploited in the design of 3D-printed soft actuators; they are capable of providing large bending deformation and thus gripping capabilities, under pressurized air (Patel et al., 2017). The use of lattice structures has also been recently exploited in the development of soft-robotic grippers: in (Kaur and Kim, 2019) auxetic joints and a cellular skeleton are used to enable the design of compliant robotic fingers.

Focusing the discussion on the 3D-printed artificial hands, it is worth underlying that, their grasping capabilities, are not as good as the not 3D-printed solutions already available (Tian et al., 2017). However, researchers, have not given up the possibility to use AM technologies to develop innovative, lightweight, low-cost, easy to assemble, customizable, and reliable solutions. As amply documented in the literature, continuously, new variants of 3D-printed robotic hands are proposed (e.g. see, Xu et al., 2013; Kappassov et al., 2013; Tian et al., 2017; Cuellar et al., 2019). This paper presents a further concept. It is characterised by a simplified design since it is composed by the palm, five fingers, and the actuation. To assemble the fingers to the palm mechanical fastening is not necessary. The "tendons" controlling the movements of the fingers are printed-in-place together with the finger; this leads to a further simplification of the assembly procedure. Compliant joints are used to drive the fingers' movements. Our 3D-printed hand is equipped with an "underactuated" transmission architecture (Piazza et al., 2019): the tendons are powered by 3 servomotors controlled through an Arduino (www.arduino.cc) board. Despite these limits, the performed validation tests demonstrated that the prototype can successfully perform a number of manipulation tasks: it can grasp, hold and release objects of different shapes, dimensions and consistency.

\section{Background}

In this section, we will focus the discussion on some recent examples of 3D-printed artificial hands and in reviewing the latest developments in the field of 3D-printed soft robotic. The aim is to underline the contribution of AM technologies in the field of artificial hands.

As underlined in (Piazza et al., 2019) soft robotic is one of the recent trends in the field of artificial hands design: flexible materials represent a valid alternative to rigid joints. In (Holland et al., 2014), a soft robotics toolkit is described to support researchers in the whole development process of soft robotic devices. In this toolkit, AM technologies are used for prototyping the moulds for fabricating the flexible soft actuator. The work of Hao et al. (2017) is focused on exploring the geometric parameters of the actuator chamber as well as the material properties that mostly influence the bending behaviour of the actuator when deflated. Hence, in the design of such devices, materials properties play an important role in enabling large deformations, but also in influencing the grasping force (Agarwal et al., 2018). The rapid evolution of AM processes and materials has allowed optimising the development of soft robotic actuators at two-level: by replacing the moulding/casting process with 3Dprinted actuators and by designing new materials capable of large deformations. The work of Patel et al. (2017) presents a family of highly stretchable photocurable elastomers that could be used as a valid alternative to silicon rubber. The experimental tests demonstrated that this family of materials guarantee not only large elastic deformations but also good mechanical performances under fatigue cycles. This is a fundamental requirement for industrial applications. Another example of photocurable elastomer, for 3D-printing soft pneumatic actuators, is described in (Ge et al., 2018). The authors used a Digital Light Processor (DLP) printer to cure a mix of a photocurable elastomer with a photo absorber to enable large deformations under relatively low pressure. Their process also guarantees a good degree of design scalability, i.e. the possibility to print, efficient and precise soft pneumatic macro- and micro-actuators. Truby et al. (2019) explored how to fabricate multi-degree-offreedom and sensorised soft actuators. To reach this target, the authors used the so-called embedded3D printing (e-3DP) method which enables the printing of strain sensors directly embedded into elastomeric layers (see also Muth et al., 2014). Further advancements, thanks to AM technologies, are proposed by $\mathrm{Ge}$ et al. (2016). The authors developed a proof-of-concept and 3D-printed shape memory polymers microgripper. An external stimulus (a heating source) is used to activate the gripper 
from its AS-BUILT condition without the need of the pneumatic actuation. Besides, the multi-material printing process allows this micro-gripper having a variable stiffness.

This overview highlights the fundamental role played by AM technologies and materials in advancing the research in the field of soft robotic solutions. Concerning the design of artificial hands, AM technologies have played a fundamental role in simplifying the design, fabrication, and the assembly phases. For example, the work of Xu et al. (2013) describes a 3D-printed hand designed by implementing a LEGO-like approach: "snap-on" joints are used to assemble the modules of each finger unit. A cable-driven system is used to control the 4 DoF of each finger. Another example of low-cost underactuated 3D-printed robotic hands is described in (Kapassov et al., 2013). The palm, the fingers and the rubber pudding of their hand are printed in-place to simplify the assembly phase and reduce its weight because, for example, fasteners are not needed. It uses 4 servomotors and again, a cable-driven system (five tendons) to control the fingers' movement. This 3D-printed hand is also sensorised to enable smart manipulation tasks through, for example, object detection and recognition. Another interesting version of lightweight, low-cost and cable-driven 3D-printed robotic hand is described in (Tian et al., 2017). This artificial hand was designed to reduce as much as possible the assembly phases. This target is reached using joints based on an interlocking strategy. Besides, a "ball joint" mechanism is used to assemble each finger to the palm. The 3D-printed robotic hand described by Cuellar et al. (2019), has been conceived to be a hand prosthesis for developing countries. It does not require post-assembly phases except for the snap-fitting of the palm for covering the mechanism. The fingers' movements are enabled by leaf springs printed in PLA as the compliant mechanism.

Finally, it is worth mentioning another relevant work (Catalano et al., 2014) which implements the concept of synergies to simplify the hand design. This concept, introduced by neuroscientists (Catalano et al., 2014; Santello et al., 2016), is based on the assumption that the complex movements of the hand sensorimotor system is instead controlled through a specific set of motor primitives through which it is possible to generate different and coherent motion patterns (Catalano et al., 2014). This approach together with an optimal design of the joints (they are compliant rolling-contact joints) and of the elastic ligaments enabled Catalano et al. (2014) to design an underactuated cable-driven (i.e. the tendons) hand which has 19 joints and only one actuator (Santello et al., 2016). The authors mention that such innovative design of joints and ligaments has also allowed exploiting the potential of currently available material deposition techniques.

Starting from this overview, in this paper, we describe an innovative concept of underactuated 3Dprinted hand. The novelty of the proposed simplified design is related to how the fingers, the joints and the tendons were conceived: they are made of the same polymeric material and printed together. This approach can further simplify the hand design.

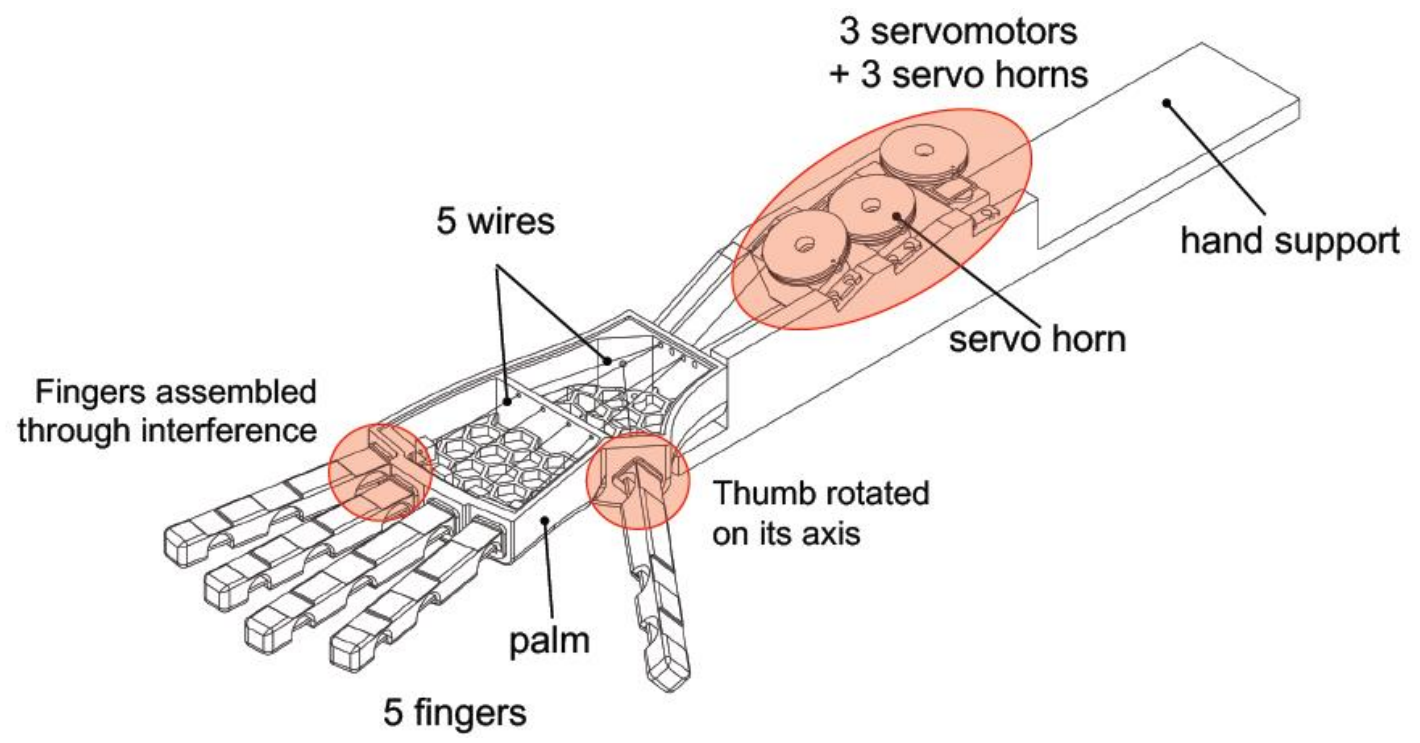

Figure 1. The assembly view of our underactuated robotic hand 


\section{System design}

In this Section, the design process of our 3D-printed underactuated hand is discussed, starting from the joints and ligaments up to the final layout and the actuation. A complete view of the prototype is provided in Figure 1. As anticipated, it consists of the following elements: 5 fingers, 5 wires for controlling the fingers movements, 1 palm, 3 servomotors, 3 servo horns and 1 support. The hand was 3D-printed using an Ultimaker 3 (2019) machine and the following materials: the Ultimaker TPU95A (2019) White for the fingers and the tendons; the Ultimaker CPE (2019) Blue for the palm, and the Ultimaker PVA (2019) as the support material. Further design and prototyping details are provided in Sections 3.1, 3.2, and 3.3.

\subsection{The joints and the tendons}

Among the available compliant joints (Trease et al., 2005) we selected the planar notch joint (Figure 2) for the following reasons: (1) it allows rotation on one axis only; (2) it allows a compact design; (3) it provides stiffness to the joint. An analytical model of a compliant joint can be derived for the simple case of linear elastic model which is however not valid in case of large deformations. Despite this limitation, the linear model can be used to quickly compare different finger's geometries, materials and configurations in terms of relative performance (Agarwal et al., 2018). A hyperelastic material model can be used such as the Yeoh's model (Renaud et al., 2009) to obtain more reliable results; this model can be used to simulate isotropic incompressible rubber-like materials such as the Ultimaker TPU95A (2019) which was used to print both the finger and the tendon. It has been demonstrated to fit various modes of deformation using the data obtained from only uniaxial tension tests (Renaud et al., 2009). However, for further details concerning the simulation of soft components see the work of Holland et al. (2014).

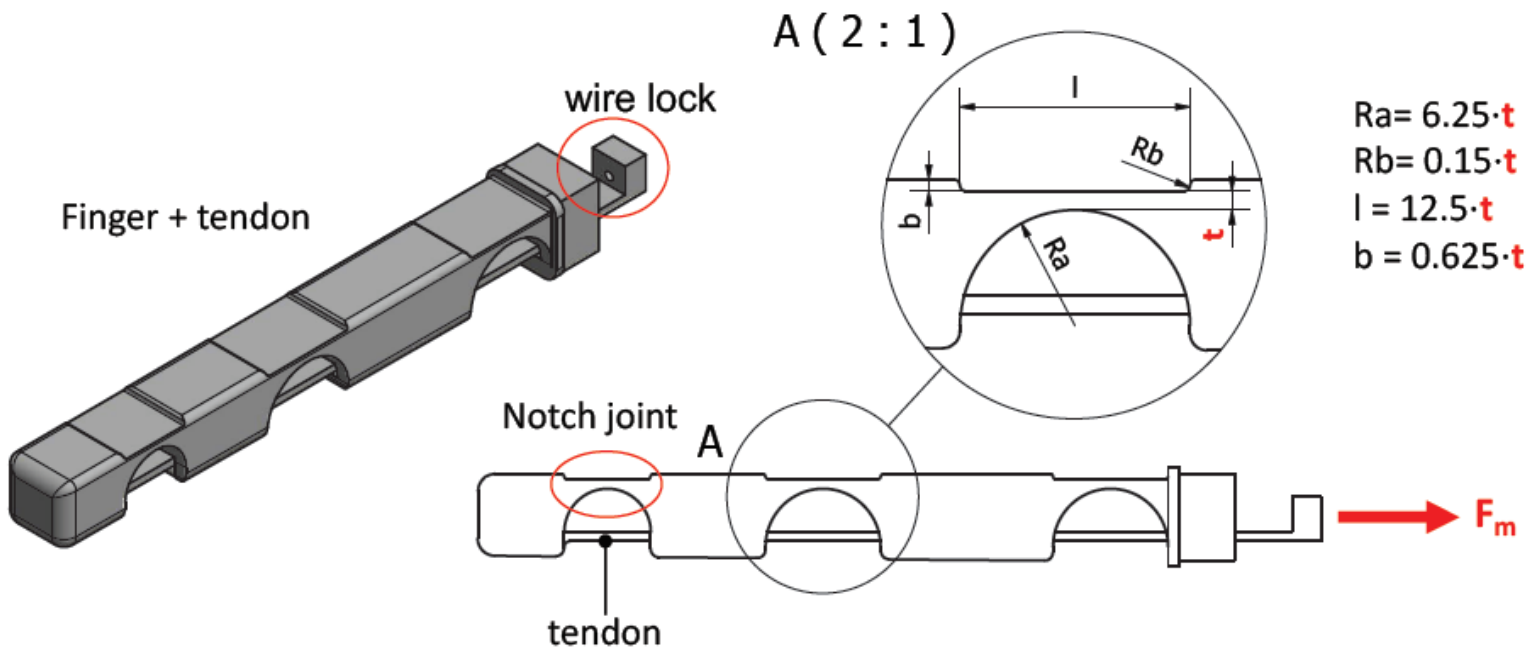

Figure 2. The parameterisation of the notch joint

The joint stiffness depends on its geometry; it increases with its height and width, while it decreases with the length. We decided to parameterise the joint geometry, as represented in Figure 2 , and by considering a unique variable, i.e., " $t$ " (Figure 2), that is the thinnest thickness of the joint (Mutlu et al., 2016). The final joint design is obtained through an iterative process, and by testing the joint deformation through a Finite Element Analysis (FEA) simulation. As shown in Figures 1 and 2, the joint actuation is performed through a tendon controlled by a motor (Fm is the force exerted by the servomotor by means of the wire, see Figure 2). The release of the tendon is automatic thanks to the elastic effect forcing the finger to go back to its original position. The Ultimaker TPU95A was selected because of its elasticity and intrinsic damping properties. The simulations were performed using the Abaqus software (www.3ds.com) while the material properties were retrieved from the datasheet (Ultimaker TPU95A, 2019). The simulations demonstrated to correctly forecast the real behaviour of the compliant joint, while the values of 
the forces, as expected, differed from the real conditions. A comparison between the simulation and the real joint is shown in Figure 3.
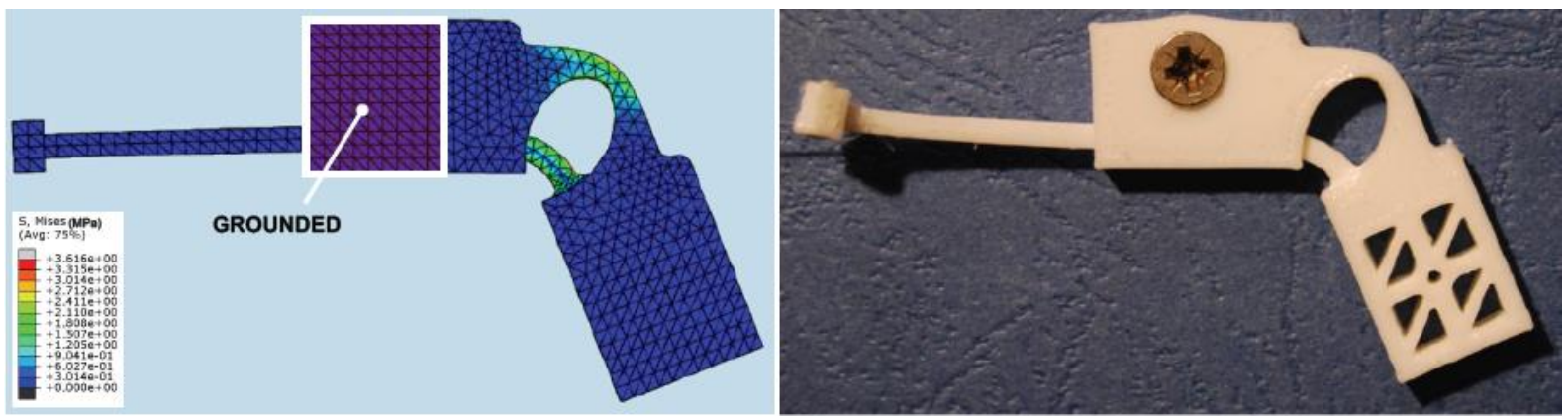

Figure 3. The simulations vs the real joint deformation; The $2 \mathrm{D}$ lattice structure on the real sample has been used to reduce the material needed for the testing

\subsection{The fingers and the palm}

Once optimised the joint shape, the finger was obtained by connecting the phalanges through these joints (Figure 4). The tendon is 3D-printed with the finger, and by using a support material (Ultimaker PVA, 2019) to avoid that these two parts get stuck during the printing. This design allowed a straightforward definition of the kinematic model of the finger's movement, as shown in Figure 4.
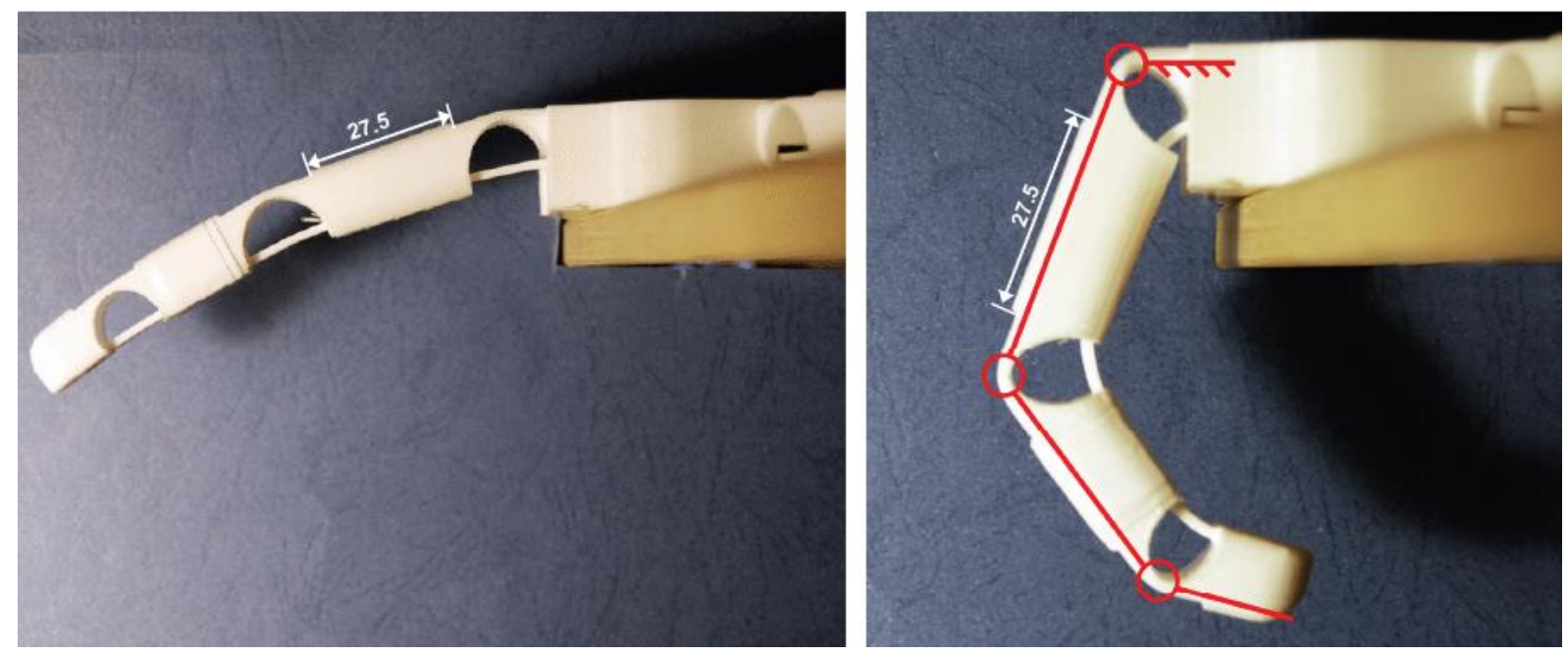

Figure 4. The kinematic model of the finger; The finger represented in this image is the index

The phalanges length was selected by comparing data of human fingers and already available robotic hands (Malvezzi et al., 2015). However, to find the proper dimensions for different fingers and their correct orientation with respect to the palm, the Syngrasp MATLAB Toolbox was used to simulate the kinematics. The tool allows to easily define the model of a hand using Denavit-Hartenberg (DH) parameters, to compute grasping tasks and to move the hand according to different synergies (Malvezzi et al., 2015; Gabiccini et al., 2011). This toolbox was used to find the best hand's configuration from a kinematic point of view. Indeed, the design of the hand was based on the grasping task, and it is inspired by the first two synergies, through which the $80 \%$ of the grasping tasks can be exploited (Santello et al., 1998; Catalano et al., 2014). Two kinematic conditions modelled through Syngrasp are provided in Figure 5. Each finger's joint provides only one DoF, enabling the flexions and extensions, while abductions and adductions were not considered. For this reason, fingers are placed with a fixed abduction/ adduction angle with respect to the palm. The three joints of each finger are underactuated through a single tendon (see Figures 2 and 4). 

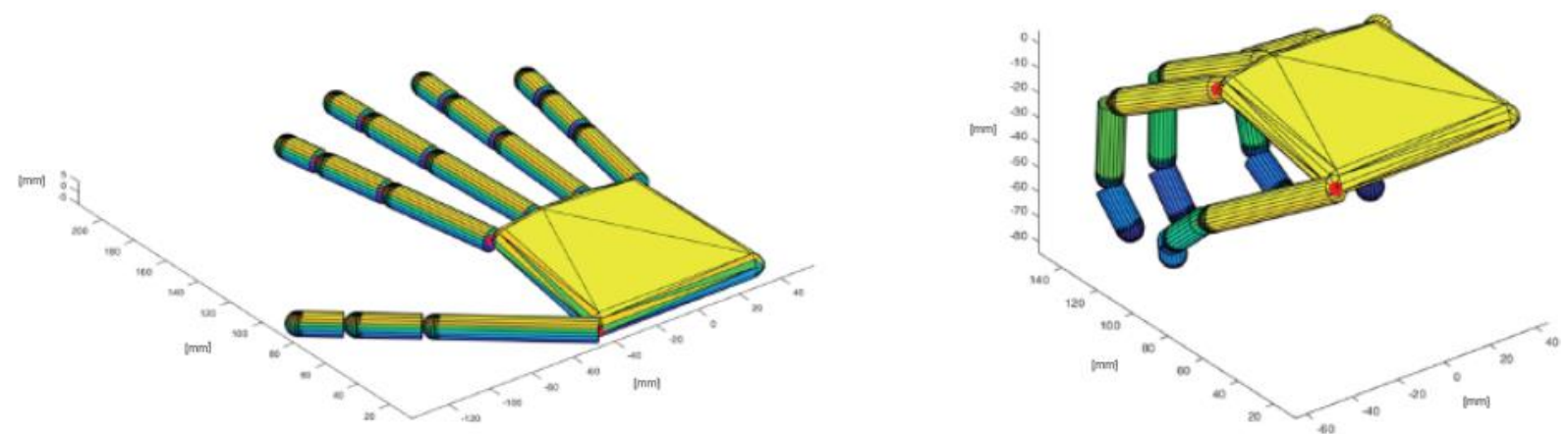

Figure 5. A grasping task (on the right) and a flat condition (on the left) of the hand simulated with the Syngrasp MATLAB Toolbox

The design parameters used in the Syngrasp MATLAB toolbox were linked to the parametric CAD models of the palm and the fingers. Kinematic simulations were also performed within the CAD environment to immediately tests the new hand's configurations before 3D-printing the final one. The whole design process is summarised in Figure 6 while the main dimensions of the hand are provided in Figure 7.

(1)

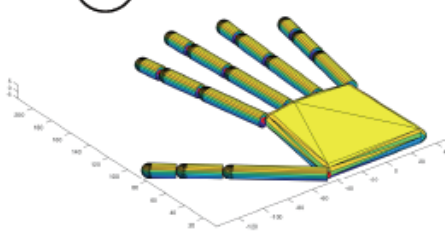

Hand model based on the Syngrasp MATLAB Toolbox
(2)

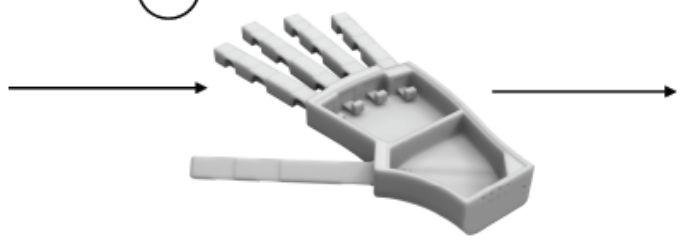

Parametric models of the palm and fingers within Autodesk Inventor
(3)

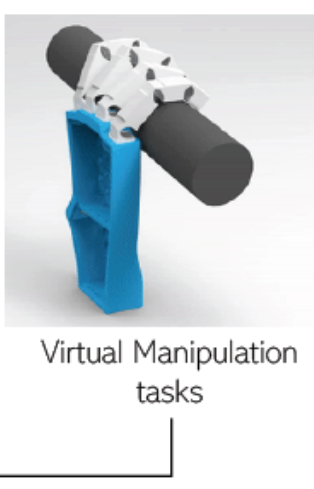

Figure 6 . The design workflow of the fingers and the palm
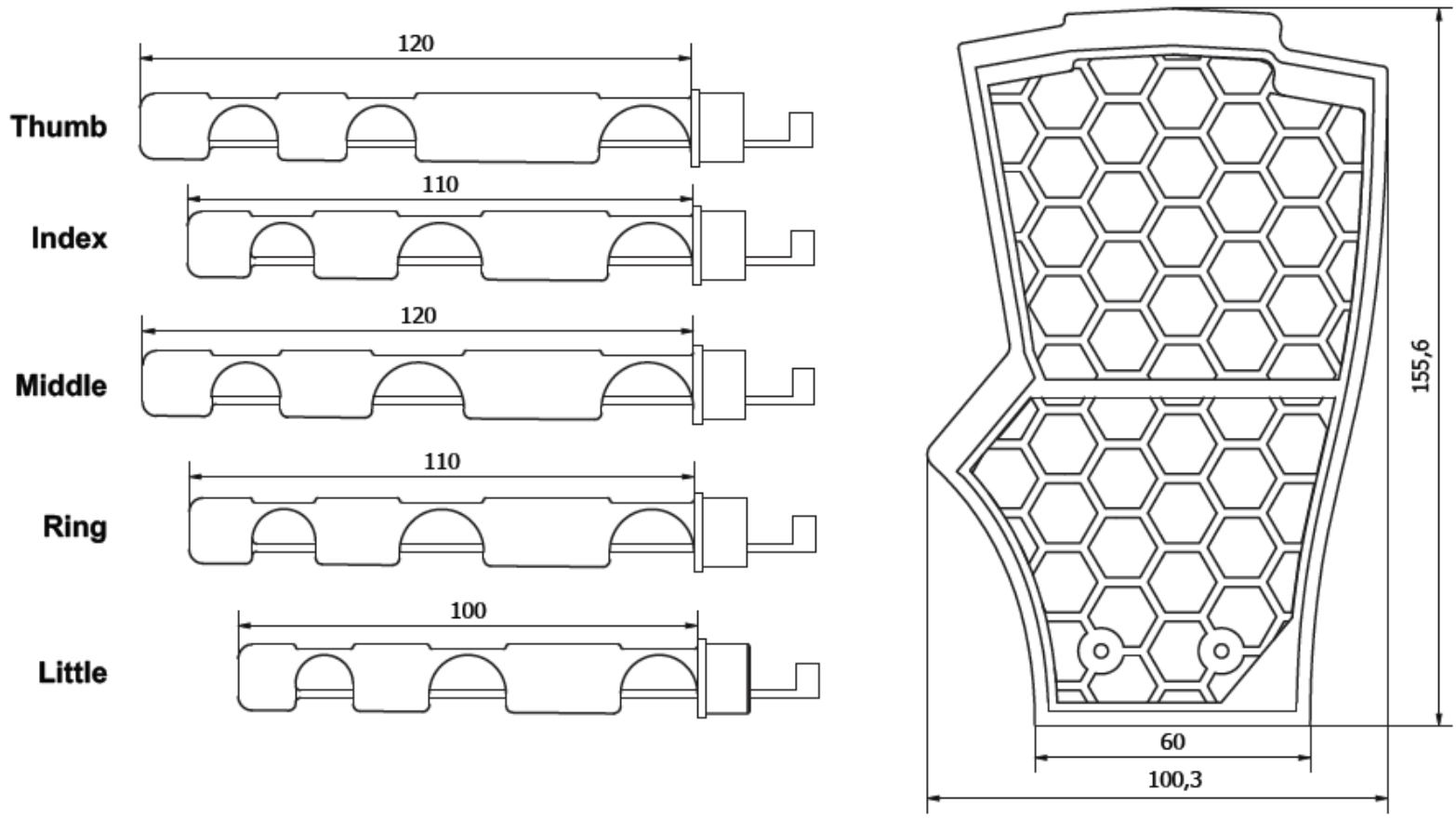

Palm

Figure 7. Main hand's dimensions 


\subsection{Tip force computation and final prototype}

The computation of the theoretical finger's strength basically focuses on the condition in which the finger is fully extended (Figure 8), that is considered as a limit case. According to this schematization, the tendon creates a moment on each joint in the finger. The first knuckle is the farthest from the applied force; hence, it can be assumed that, the force at this joint, limits the liftable load. Using a simple moment balance the maximum liftable load at the tip can be computed as (see Figure 8)

$$
\mathrm{F}_{\text {tip }}=\left(\mathrm{F}_{\mathrm{m}} \cdot(\mathrm{b}+\mathrm{t} / 2)\right) / \mathrm{a}
$$

where $F_{m}$ is the linear force pulling the tendon (see also Figure 2), obtained as

$$
\mathrm{F}_{\mathrm{m}}=\mathrm{C}_{\mathrm{m}} / \mathrm{r}_{\text {HORN }}
$$

being $\mathrm{C}_{\mathrm{m}}$ the motor torque and $\mathrm{r}_{\text {HORN }}$ the servo horn radius (Figure 1). It is worth underlying that this is the worst situation. Once the finger start closing, the equivalent moment exerted on the knuckle joint by the tip force inevitably decreases of the "a" value (Figure 8 ).

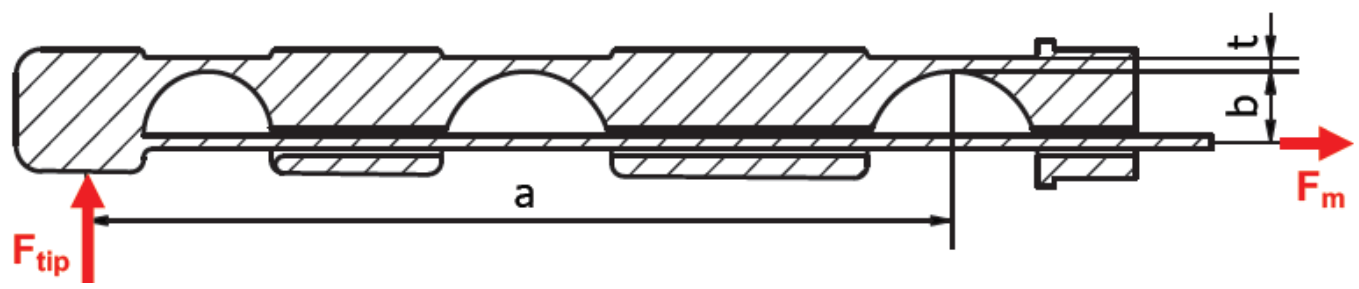

Figure 8. How the maximum tip force was calculated: the finger is completely extended, $F_{\text {tip }}$ is exerted at the fingertip centre while $F_{m}$ is exerted by the tendon being pulled

The axial tensile strength applied at each wire, to complete the finger ride, was experimentally investigated by fixing the finger and applying a weight on it. The results stated that at least $1.75 \mathrm{~kg}$ for each finger is required.

As anticipated, the fingers' movements are controlled through three servomotors $(3 \mathrm{~kg} \cdot \mathrm{cm} \mathrm{each})$, the first attached to index and middle fingers ("I" and "M", Figure 9), the second attached to the ring and little fingers ("R" and "L", Figure 9), and the third connected to the thumb ("T", Figure 9). For the thumb, we used the servo-motor SANWA SRM 102 (http://sanwa-denshi.com/rc/car/servo/sx112.html), while the other two servo-motors are the DATAN S1213 model (https://www.adafruit. com/product/1404).

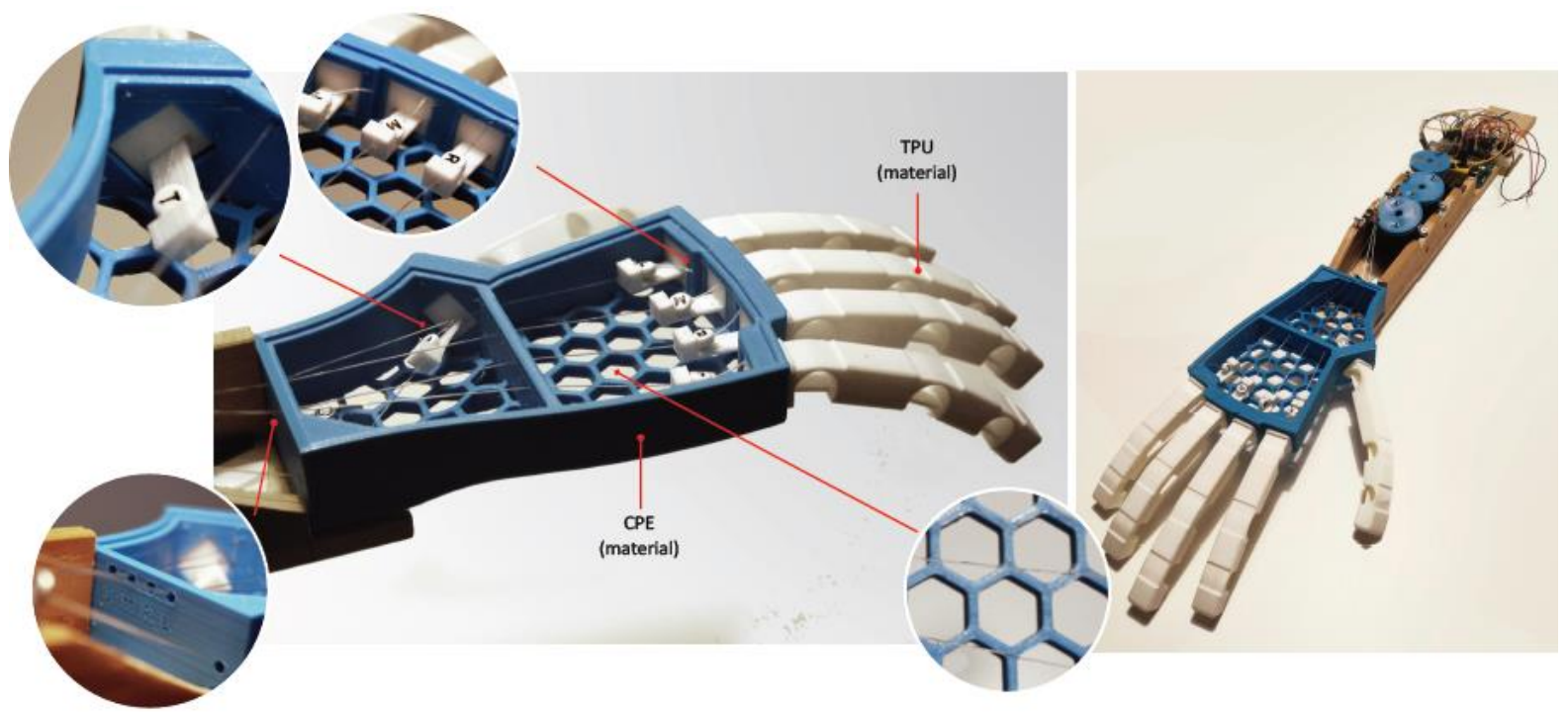

Figure 9. The final prototype of the hand 
The 3D printed horns (38 mm diameter, Figures 1 and 9), are mounted on the servos to guarantee the whole finger bending: it implies that the available motor force is approximately $1.5 \mathrm{~kg}$ each (i.e., $3 \mathrm{~kg} \cdot \mathrm{cm} /(3.8 \mathrm{~cm} / 2))$. Indeed, the three servo motors are not able to completely close the fingers. This fact has not limited the grasping tasks that our artificial hand can perform (see Section 4). Besides, the complete closure of the fingers is not necessary, since it could damage the objects. Thanks to the three servos, we can have independent movements of the thumb, the index and middle fingers, the ring and little fingers (see Section 4 for further details).

The five fingers are easily replaceable because they are assembled with interference (Figure 1) while the palm was 3D-printed using a honeycomb structure (Figure 9) to optimise printing times.

\section{Experimental validation}

Thanks to the implemented synergies, the prototype can grasp various objects, but also pinch, reaching small targets dimensions. From the experimental results, it can be observed that the hand can grasp spherical targets with a diameter in the range between $30 \mathrm{~mm}$ and $85 \mathrm{~mm}$, as shown in Figure 10. The task represented in Figure 10a was used to test 5 fingers working together on a tube having the following dimensions: $50 \mathrm{~mm}$ diameter and $230 \mathrm{~mm}$ length. The grasping was performed with the help of an operator who had to hand the object because the prototype was not able to grasp it from a flat surface. The second task (Figure 10b) refers to the lateral pinching of a small ball of 30 $\mathrm{mm}$ diameter starting from a planar surface. The third task (Figure 10c) refers to 3 fingers grasping a $45 \mathrm{~mm}$ diameter ball with the help of an operator as in the case of the first task. Finally, the last task (Figures 10d and 11) was performed on a bigger sphere (85 mm diameter) with 5 fingers grasping and catching it from a planar surface. The maximum payload is influenced by the mechanical properties of the TPU, the hand design and the servomotors' power.
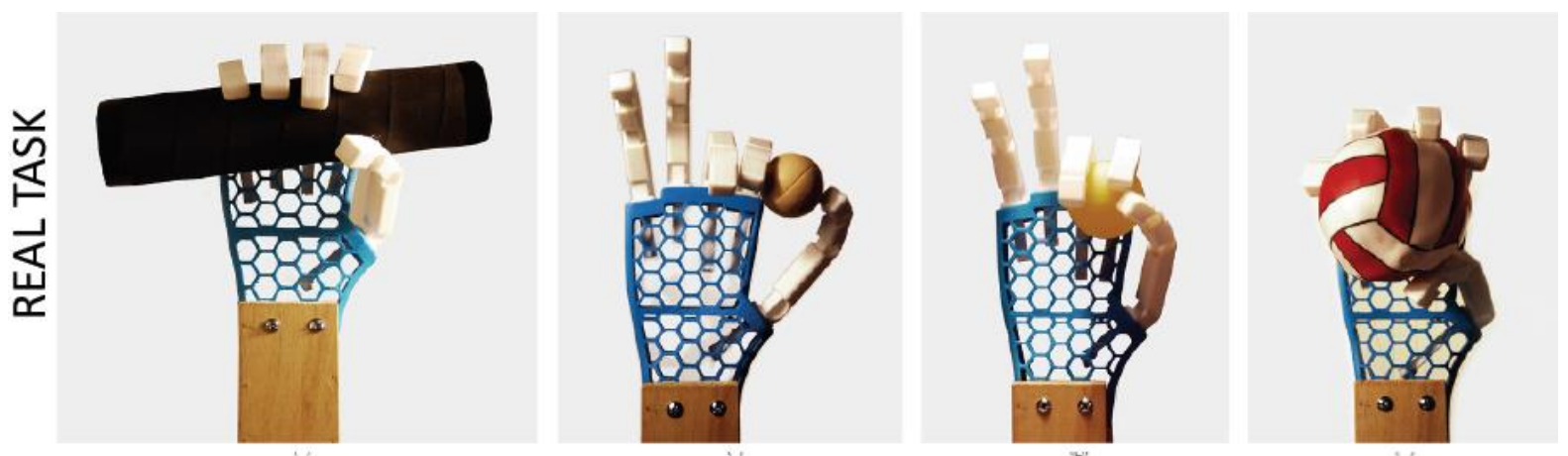

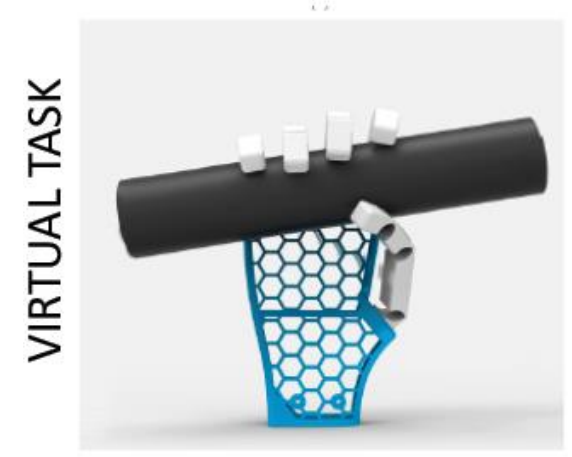

(a)

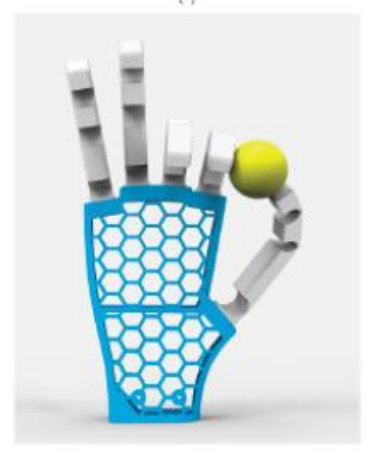

(b)

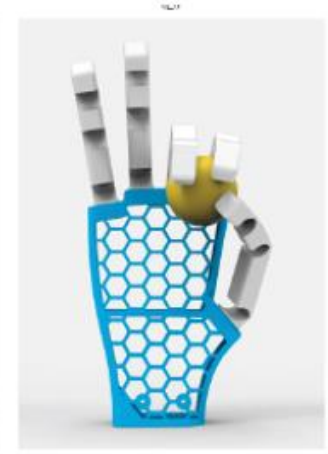

(c)

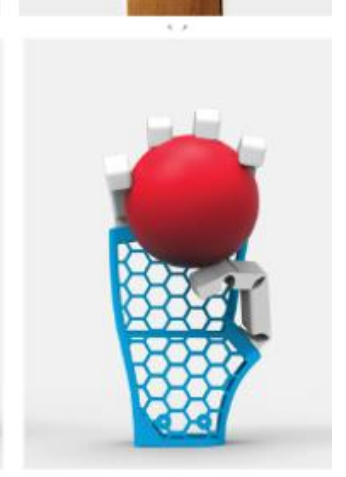

(d)

Figure 10. Comparison between the real and the virtual manipulation tasks

The lack of sensors does not allow regulating the pressure exerted on the object. A glove can be used to cover the hand, provide grip and preserve the hand from any external factors that could alter its capabilities. Finally, the fact that the prototype is not able to grasp some objects from a flat surface is a limit that can be overcome by improving the design and the control of the fingers. 


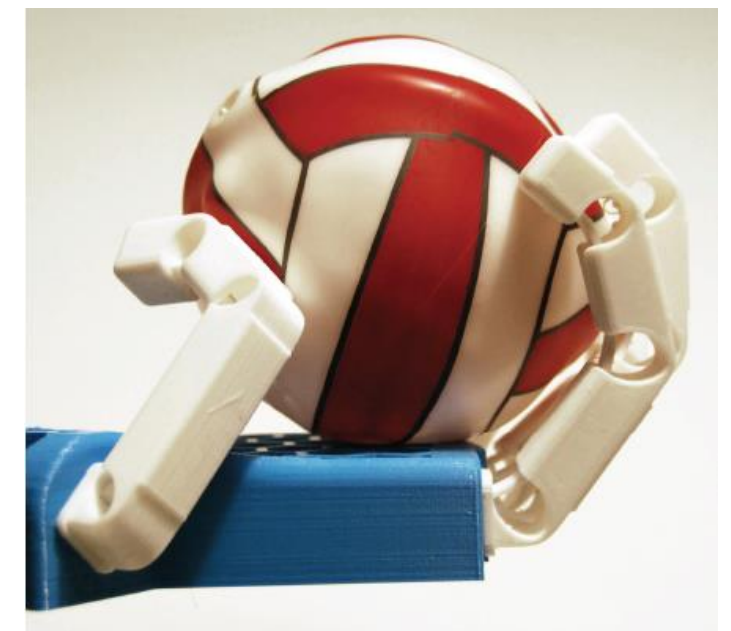

Figure 11. A lateral view of the grasping task represented in Figure 10d

\section{Conclusions}

This paper describes an innovative low-cost 3D-printed robotic hand. A compliant finger's joint was designed and optimised. A kinematic model of the hand was developed to investigate its grasping capability before prototyping it. The tendons controlling the fingers' movements are print-in-place with the fingers. This solution has further simplified the system and thus, its assembly process. The model of the joint can be further extended to estimate joint stiffness and damping, from which a lumped parameters dynamical model of the system could be obtained (Mutlu et al., 2016). More advanced joint solutions could be explored, for example, by changing the material, varying the mass spatial distribution or designing external/internal additional frames (Schumacher et al., 2015). Finally, the actuation can be further improved by embedding sensors, but also taking into account the need for minimalist solutions (Piazza et al., 2019). The prototype has some limits because it does not implement abduction and adduction movements of the fingers. Besides, in the developed model, friction-related aspects are not taken into account. Finally, more specific testing activities concerning the efficiency of the mechanisms are necessary as, for example, performed in (Cuellar et al., 2019).

\section{References}

Agarwal, A. et al. (2018), "Effects of material properties on soft gripper grasping forces", In 2018 IEEE International Conference on Soft Robotics (RoboSoft), pp. 437-442. https://doi.org/10.1109/ROBOSOFT .2018 .8405365

Bhatia, A., Johnson, A.M. and Mason, M.T. (2019), "Direct Drive Hands: Force-Motion Transparency in Gripper Design", Proceedings of the Robotics: Science and Systems, Freiburg, Germany, pp. 22-26. https://doi.org/10.15607/RSS.2019.XV.053

Catalano, M.G., et al. (2014), "Adaptive synergies for the design and control of the Pisa/IIT SoftHand", The International Journal of Robotics Research, Vol. 33 No. 5, pp. 768-782. https://doi.org/10.1177\% 2F0278364913518998

Cuellar, J.S. et al. (2019), "Functional evaluation of a non-assembly 3D-printed hand prosthesis", Proceedings of the Institution of Mechanical Engineers, Part H: Journal of Engineering in Medicine, Vol. 233 No. 11, pp. 1122-1131.https://doi.org/10.1177\%2F0954411919874523

Gabiccini, M. et al. (2011), "On the role of hand synergies in the optimal choice of grasping forces", Autonomous Robots, Vol. 31 No. 2-3, pp. 235-252. https://doi.org/10.1007/s10514-011-9244-1

Ge, L. et al. (2018), "A digital light processing 3D printer for fast and high-precision fabrication of soft pneumatic actuators”, Sensors and Actuators A: Physical, Vol. 273, pp. 285-292. https://doi.org/10.1016/ j.sna.2018.02.041

Ge, Q. et al. (2016), "Multimaterial 4D printing with tailorable shape memory polymers", Scientific reports, Vol. 6, p. 31110. https://doi.org/10.1038/srep31110

Hao, Y. et al. (2017), "Modeling and experiments of a soft robotic gripper in amphibious environments", International Journal of Advanced Robotic Systems, Vol. 14 No. 3, p. 1729881417707148. https://doi.org/ $10.1177 \%$ 2F1729881417707148 
Holland, D.P. et al. (2014), "The soft robotics toolkit: Shared resources for research and design", Soft Robotics, Vol. 1 No. 3, pp. 224-230. https://doi.org/10.1089/soro.2014.0010

Kappassov, Z. et al. (2013), "Semi-anthropomorphic 3D printed multigrasp hand for industrial and service robots", In 2013 IEEE International Conference on Mechatronics and Automation, pp. 1697-1702. https://doi.org/10.1109/ICMA.2013.6618171

Kaur, M. and Kim, W.S. (2019), "Toward a Smart Compliant Robotic Gripper Equipped with 3D-Designed Cellular Fingers", Advanced Intelligent Systems, Vol. 1 No. 3, p. 1900019. https://doi.org/10.1002/aisy.201900019

Malvezzi, M. et al. (2015), "Syngrasp: A matlab toolbox for underactuated and compliant hands", IEEE Robotics \& Automation Magazine, Vol. 22 No. 4, pp. 52-68. https://doi.org/10.1109/MRA.2015.2408772

Matsas, E., Vosniakos, G.C. and Batras, D. (2018), "Prototyping proactive and adaptive techniques for humanrobot collaboration in manufacturing using virtual reality", Robotics and Computer-Integrated Manufacturing, Vol. 50, pp. 168-180. https://doi.org/10.1016/j.rcim.2017.09.005

Muth, J.T. et al. (2014), "Embedded 3D printing of strain sensors within highly stretchable elastomers", Advanced Materials, Vol. 26 No. 36, pp. 6307-6312. https://doi.org/10.1002/adma.201400334

Mutlu, R. et al. (2016), "3D printed flexure hinges for soft monolithic prosthetic fingers", Soft Robotics, Vol. 3 No. 3, pp. 120-133. https://doi.org/10.1089/soro.2016.0026

Patel, D.K. et al. (2017), "Highly stretchable and UV curable elastomers for digital light processing based 3D printing", Advanced Materials, Vol. 29 No. 15, p. 1606000. https://doi.org/10.1002/adma.201606000

Piazza, C. et al. (2019), "A century of robotic hands", Annual Review of Control Robotics, and Autonomous Systems, Vol. 2, pp. 1-32. https://doi.org/10.1146/annurev-control-060117-105003

Renaud, C. et al. (2009), "The Yeoh model applied to the modeling of large deformation contact/impact problems", International Journal of Impact Engineering, Vol. 36 No. 5, pp. 659-666. https://doi.org/ 10.1016/j.ijimpeng.2008.09.008

Santello, M., Flanders, M. and Soechting, J.F. (1998), "Postural hand synergies for tool use", Journal of Neuroscience, Vol. 18 No. 23, pp. 10105-10115. https://doi.org/10.1523/JNEUROSCI.18-23-10105.1998

Santello, M. et al. (2016), "Hand synergies: integration of robotics and neuroscience for understanding the control of biological and artificial hands", Physics of life reviews, Vol. 17, pp. 1-23. https://doi.org/ 10.1016/j.plrev.2016.02.001

Schumacher, C. et al. (2015), "Microstructures to control elasticity in 3D printing", ACM Transactions on Graphics (TOG), Vol. 34 No. 4, p. 136. https://doi.org/10.1145/2766926

Ten Kate, J., Smit, G. and Breedveld, P. (2017), "3D-printed upper limb prostheses: a review”, Disability and Rehabilitation: Assistive Technology, Vol. 12 No. 3, pp. 300-314. https://doi.org/10.1080/17483107. 2016.1253117

Tian, L. et al. (2017), "The making of a 3D-printed, cable-driven, single-model, lightweight humanoid robotic hand", Frontiers in Robotics and AI, Vol. 4, p. 65. https://doi.org/10.3389/frobt.2017.00065

Trease, B.P., Moon, Y.M. and Kota, S. (2005), "Design of large-displacement compliant joints", Journal of mechanical design, Vol. 127 No. 4, pp. 788-798. https://doi.org/10.1115/1.1900149

Truby, R.L. et al. (2019), "Soft Robotic Fingers with Embedded Ionogel Sensors and Discrete Actuation Modes for Somatosensitive Manipulation", In 2019 2nd IEEE International Conference on Soft Robotics (RoboSoft), pp. 322-329. https://doi.org/10.1109/ROBOSOFT.2019.8722722

$\mathrm{Xu}, \mathrm{Z}$., Kumar, V. and Todorov, E. (2013), “A low-cost and modular, 20-DOF anthropomorphic robotic hand: design, actuation and modeling", In 2013 13th IEEE-RAS International Conference on Humanoid Robots (Humanoids), pp. 368-375. https://doi.org/10.1109/HUMANOIDS.2013.7030001

Ultimaker 3, (2019), https://ultimaker.com/3d-printers/ultimaker-3, (accessed 06.11.2019)

Ultimaker CPE, (2019), https://ultimaker.com/en/resources/49914-cpe, (accessed 06.11.2019)

Ultimaker PVA, (2019), https://ultimaker.com/en/resources/49919-pva, (accessed 06.11.2019)

Ultimaker TPU95A, (2019), https://ultimaker.com/en/resources/49917-tpu-95a, (accessed 06.11.2019) 